Article

\title{
An Onboard Vision-Based System for Autonomous Landing of a Low-Cost Quadrotor on a Novel Landing Pad
}

\author{
Xuancen Liu, Shifeng Zhang *, Jiayi Tian and Longbin Liu \\ College of Aerospace Science and Engineering, National University of Defense Technology, Changsha 410073, China; \\ liuxuancen14@nudt.edu.cn (X.L.); tianjiayi13@nudt.edu.cn (J.T.); liulongbin@nudt.edu.cn (L.L.) \\ * Correspondence: zhang_shifeng@hotmail.com
}

Received: 20 August 2019; Accepted: 27 October 2019; Published: 29 October 2019

check for updates

\begin{abstract}
In this paper, an onboard vision-based system for the autonomous landing of a low-cost quadrotor is presented. A novel landing pad with different optical markers sizes is carefully designed to be robustly recognized at different distances. To provide reliable pose information in a GPS (Global Positioning System)-denied environment, a vision algorithm for real-time landing pad recognition and pose estimation is implemented. The dynamic model of the quadrotor is established and a system scheme for autonomous landing control is presented. A series of autonomous flights have been successfully performed, and a video of the experiment is available online. The efficiency and accuracy of the presented vision-based system is demonstrated by using its position and attitude estimates as control inputs for the autonomous landing of a self-customized quadrotor.
\end{abstract}

Keywords: onboard vision; micro aerial vehicle (MAV); monocular camera; autonomous landing; low-cost sensors

\section{Introduction}

Over the past decade, significant progress has been achieved toward the automation of aerial robotic vehicles and related technology, leading to a variety of potential applications such as emergency response, traffic monitoring, inspection of power cables, package delivery, etc. [1,2]. micro aerial vehicles (MAVs) such as quadrotors are potentially able to move flexibly and explore efficiently in unforeseeable 3D environments with complex terrains, which is almost impossible for ground robots while executing time-sensitive missions. However, because of the limited payload and onboard battery endurance, micro quadrotors have a relatively shorter flight time than fixed-wing aircrafts, mostly ranging between 8 and $25 \mathrm{~min}$. Consequently, micro quadrotors are programmed to land on a specific platform and get recharged periodically for operations that cover large areas. In such a scenario, how to precisely and robustly land an autonomous quadrotor on a ground platform is still a challenging task of MAVs, considering that the onboard processing power is strictly limited and sensors are micro.

As the most difficult and risky phase in flight, autonomous landing requires robust recognition of the landing platform and accurate measurement of the MAV's movement with respect to the ground target. So far, it is still difficult to accurately estimate the relative position between the MAV and the landing platform unless using 3D light detection and ranging (Lidar) sensors or a differential global positioning system (DGPS), which are heavy and unaffordable [3-5]. For this reason, novel sensors are studied in many research works toward autonomous landing. [6] presented a low-cost visual tracking system for the hovering control of a Hummingbird quadrocopter by using a Wii remote infrared (IR) camera and a pattern of four infrared spots fixed on the landing pad. In [7], a low-cost solution based on a monocular camera was implemented for the autonomous takeoff, hovering, and landing of a MAV. By using projective geometry [8-10], the 6 degrees of freedom (DOF) pose of the quadrotor relative 
to a typical landing pad (the letter " $\mathrm{H}$ " surrounded by a circle) could be accurately estimated from image streams. In [11], a complete ship deck simulation for the autonomous landing of a helicopter on ships was proposed by using a single downward-looking camera and a moving platform with helipad marks. Another approach inspired by the behavior of insects used optical flow as feedback for MAVs in visual servo control [12], since it provided relative velocity to the dynamic environment [13]. Herissé et al. [14] presented a nonlinear controller for a vertical takeoff and landing (VTOL) unmanned aerial vehicle (UAV) that enabled hovering above and landing on a moving platform, by exploiting measurement of the average optical flow.

Compared with the sensors mentioned above, monocular cameras passively receive environmental information and have an inherent potential for object recognition tasks, while still being lightweight, low cost, and computationally efficient [15]. Unlike stereo cameras with limited baselines, monocular cameras are able to keep functioning even if the object is detected at a large distance. Considering that MAVs have limited payload and computational capability, monocular vision is comparably more attractive for autonomous landing and extended applications. In [16], AprilTag markers [17] were recognized by a single forward-looking camera mounted on the AR.Drone micro UAV, and state estimation was achieved based on a delayed-state extended Kalman filter (EKF) in GPS-denied situations. [18] presented an integrated visual detection system with a standard field of view camera lens and a fisheye lens occasionally used to capture faraway or highly close targets.

Due to the importance of autonomous localization and navigation, the issue of simultaneous localization and mapping (SLAM) has triggered a lot of interest in the research community, with the wide use of monocular vision. In [19], a model of the vision inertial absolute navigation system (VIANS) was established, and estimation of absolute navigation information was achieved based on the presented EKF and unscented Kalman filter (UKF). Shen et al. [20] and [21] presented a monocular visual-inertial system (VINS-Mono) for the 6DOF state estimation of UAVs, in which only a monocular camera and a low-cost inertial measurement unit were utilized. The proposed visual-inertial odometry (VIO) obtained high accuracy based on the real-time fusion of pre-integrated inertial measurement units (IMU) data and feature observations, and then the relocalization process started to eliminate accumulative drifts. In [22], a versatile visual marker-based multi-sensor fusion estimator was presented, which combined a variable optional number of sensors and positioning algorithms in a loosely coupling fashion. The results of estimation showed high accuracy in real experiments, controlling a quadrotor equipped with an IMU and an RGB camera.

In order to recognize the landing platform and precisely estimate the 6DOF pose with images captured by the onboard camera, artificial markers are widely used in autonomous landing tasks, especially square-based fiducial markers such as Matrix [23], ARTag [24], ARToolKit [25], ARToolKitPlus [26], AprilTag [17], and ArUco [27]. These markers are generally encoded by an inner binary code in order to be uniquely identified. With error detection and correction, the four corners of the identified marker can be used as reference points to estimate the camera pose by solving the Perspective-n-Point (PnP) problem [28-33], given that the camera is properly calibrated. Amongst these fiducial markers proposed in recent papers, ArUco markers, presented by Garrido-Jurado et al, have gained popularity in visual servo systems [34]. By using the ArUco library supported by OpenCV, it is not difficult to generate configurable dictionaries of markers and make a $\mathrm{C}++$ program capable of identifying and localizing those markers within the predefined dictionary. However, of all the implemented marker-based autonomous systems, it is still a challenging task to acquire accurate pose estimates by using low-cost cameras with large image noises in the movement of a MAV. When getting much closer to the landing platform, the camera may lose part of the marker due to its restricted field of view (FOV) and rotation movement, so that the estimates of the camera pose will be extremely unreliable.

In this paper, motivated by the existing challenges mentioned above, an onboard monocular vision algorithm for the $6 \mathrm{DOF}$ pose estimation of a MAV is proposed by utilizing a consumer camera. A novel landing pad is designed with a predefined configuration of several ArUco markers surrounded 
by a circle, which observably improve the detection range and the robustness of recognition. With a dynamic weighed mean filter, a fusion estimation method is presented for a more accurate 6DOF pose with respect to the landing pad. Then, the relative position, velocity, and yaw angle are put into a cascade proportional-integral-derivative (PID) controller as input information to control the hovering flight of the MAV in GPS-denied situations, thus paving the way for autonomous landing on a specific landing pad. Based on the DJI F450 frame, an experimental quadrotor is self-customized and equipped with a low-cost camera at the price of $\$ 10$, a microcontroller using consumer sensors, and an onboard computer for image processing. Compared with measurements of external positioning devices, the presented system is demonstrated to be reliable and cost-effective for the precision landing of an autonomous MAV.

The rest of this paper is organized as follows. In Section 2, the design of the landing pad and the algorithm used to identify the pattern are described. The estimation of the camera pose relative to the landing pad is presented and a method for fusion estimation is described. In Section 3, the dynamic model of the micro quadrotor is established, and the architecture of the overall landing system is presented. Section 4 describes the setup of the experiments, and practical results are given in this part. Finally, Section 5 concludes this paper.

\section{Landing Pad Design and Vision Algorithm}

\subsection{Landing Pad Design and Recognition}

The most commonly used landing pads—such as a letter " $\mathrm{H}$ " surrounded by a circle—are not highly featured and may not be uniquely identified in a cluttered environment. False positives caused by similar shapes of other objects in the background will inevitably reduce the robustness and reliability of the vision system. Compared with other patterns, square-based fiducial markers are an efficient approach to achieve both high speed and precision, which use an inner binary code for identification and error correction. The markers chosen for the design of the landing pad are generated from the predefined dictionaries of the ArUco library in OpenCV. Each marker is composed by a black border and an inner binary matrix ( $6 \times 6$ bits), which encodes its unique identification (id).

To detect and decode an ArUco marker, the image is converted to gray-scale and segmented using a local adaptive threshold strategy at the first step. Then, a contour detection and a polygonal approximation are performed. After the perspective projection is removed by computing the homography matrix, the resulting image can be divided into a grid, and each element is assigned a value of 0 or 1 by using the Otsu thresholding method [35]. Once the binary code is extracted, the marker and its unique identification will be determined if it actually belongs to the predefined dictionary.

In [36], the proposed Fractal Marker, a novel type of marker, is built as an aggregation of squared markers of different sizes, one into another, in a recursive manner, and a method for marker detection under severe occlusions is also presented. Taking into account the latest advances in the fractal ArUco, a novel landing pad with a wider detection range is designed in this paper to achieve a better performance in autonomous landing tasks. Figure 1 presents the predefined landing pad, which consists of eight unique ArUco markers surrounded by a circle. The radius of the circle is $700 \mathrm{~mm}$, which facilitates its fast detection in the image at a large distance. The location of each marker is carefully designed, and the physical size of these markers ranges from the minimum $(29 \times 29 \mathrm{~mm})$ to the maximum $(135 \times 135 \mathrm{~mm})$, so that the desired marker can be kept within the restricted FOV of the onboard camera at different heights. It is worth mentioning that the smallest marker $(29 \times 29 \mathrm{~mm})$ is placed inside the marker $(120 \times 120 \mathrm{~mm})$ at the center of the landing pad, which is carefully designed so that the vision system will not lose all of the markers, even if the camera is very close to the landing pad. Based on this, a smaller "blind" range will be achieved. 


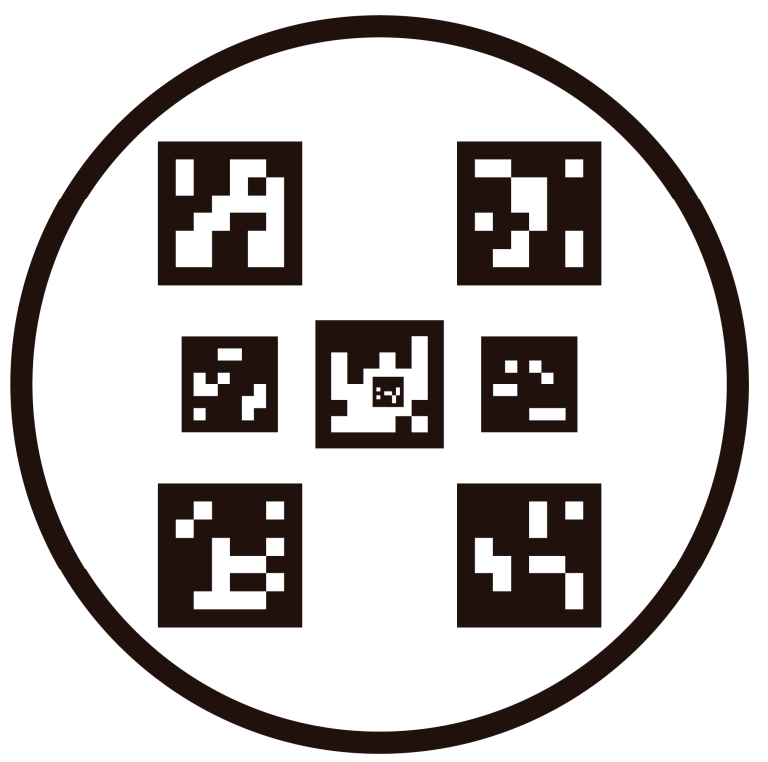

Figure 1. Configuration of the predefined landing pad.

The optimal design and configuration of the landing pad significantly improves the accuracy of recognition instead of using an unaffordable camera with high resolution. As shown in Figure 2, the bigger markers can be detected and recognized at a larger distance, and if the UAV is much closer to the landing pad, the smaller ones could still be recognized.

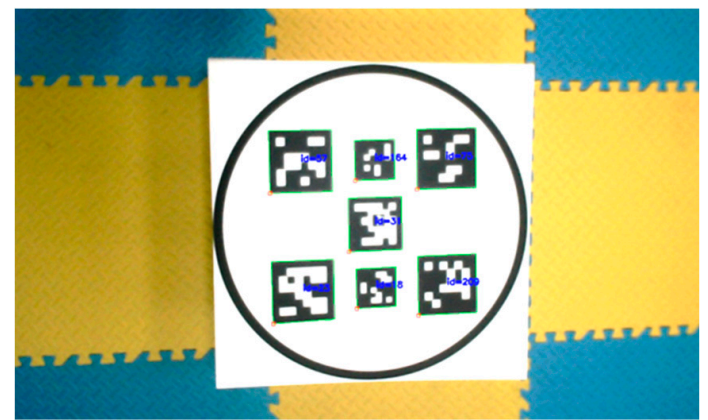

(a)

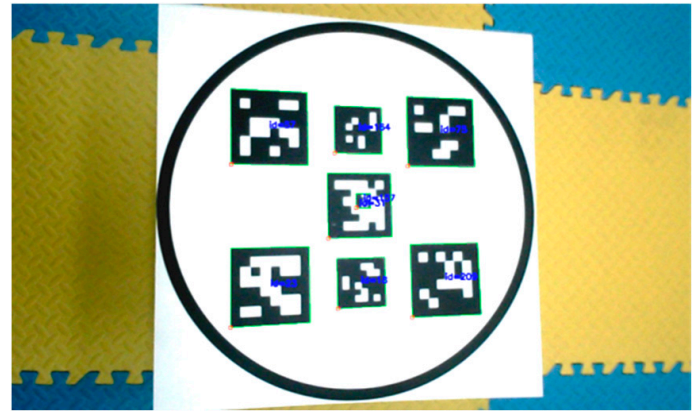

(b)

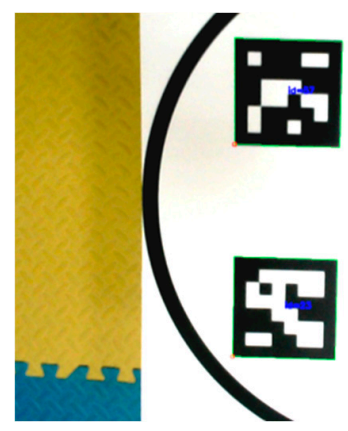

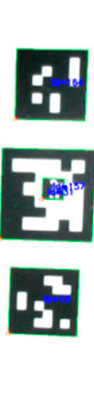

(c)
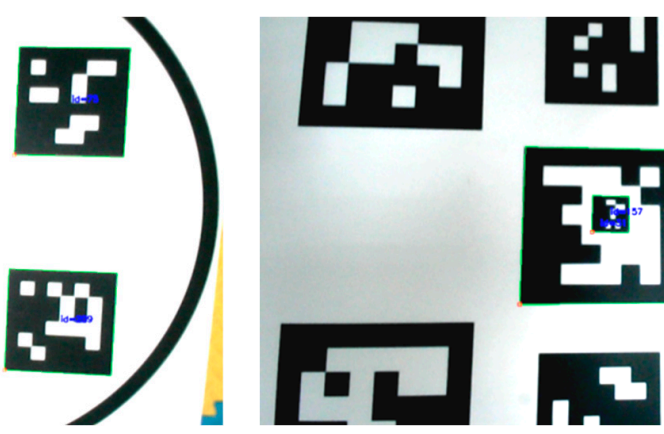

(d)

Figure 2. Markers recognized at different heights in camera view. When the camera is far away from the landing pad, the whole circle is captured in the view $(\mathbf{a}, \mathbf{b})$. With the change of attitude and height during a landing maneuver, part of the pattern moves out of the camera view (c). When the camera gets very close to the landing pad, only two markers can be recognized (d). 
To compute the relative position to the landing pad, the physical size and position of the detected marker must be known. When more than one marker is recognized, the measurement noise can be greatly reduced, and the precision can be improved by data fusion.

\subsection{Relative Pose Estimation}

The problem of solving the exterior orientation of a calibrated camera given reference 3D points and their corresponding 2D projections is commonly referred to as a Perspective-n-Point (PnP) problem, which is one of the most fundamental researches in computer vision. For large values of $\mathrm{n}$, the direct linear transformation (DLT) method is commonly used to compute the camera pose. However, due to the overlook of the intrinsic camera parameters that we are able to acquire, the DLT method is comparatively inaccurate. The clamped DLT is an alternative method that exploits the known intrinsic parameters, but the accuracy is still low. Even though some non-iterative methods such as perspective-three-point (P3P) and efficient perspective-n-point (EPnP) are relatively fast at finding an optimal solution, these are not considered because they are not especially robust in planar cases, and sometimes lead to a mirror effect. Compared with these methods, the iterative method and the homography model for planar patterns are selected to estimate the camera pose, which is followed by nonlinear optimization using the Levenberg-Marquardt algorithm to minimize the reprojection error [37].

The coordinate systems are defined in Figure 3. In the camera frame, the origin is the optic center, and $Z_{c}$ coincides with the optic axis of the camera. It is also worth mentioning that the origin of the world frame $W$ is set at the center of the landing pad, which is attached to the ground platform.

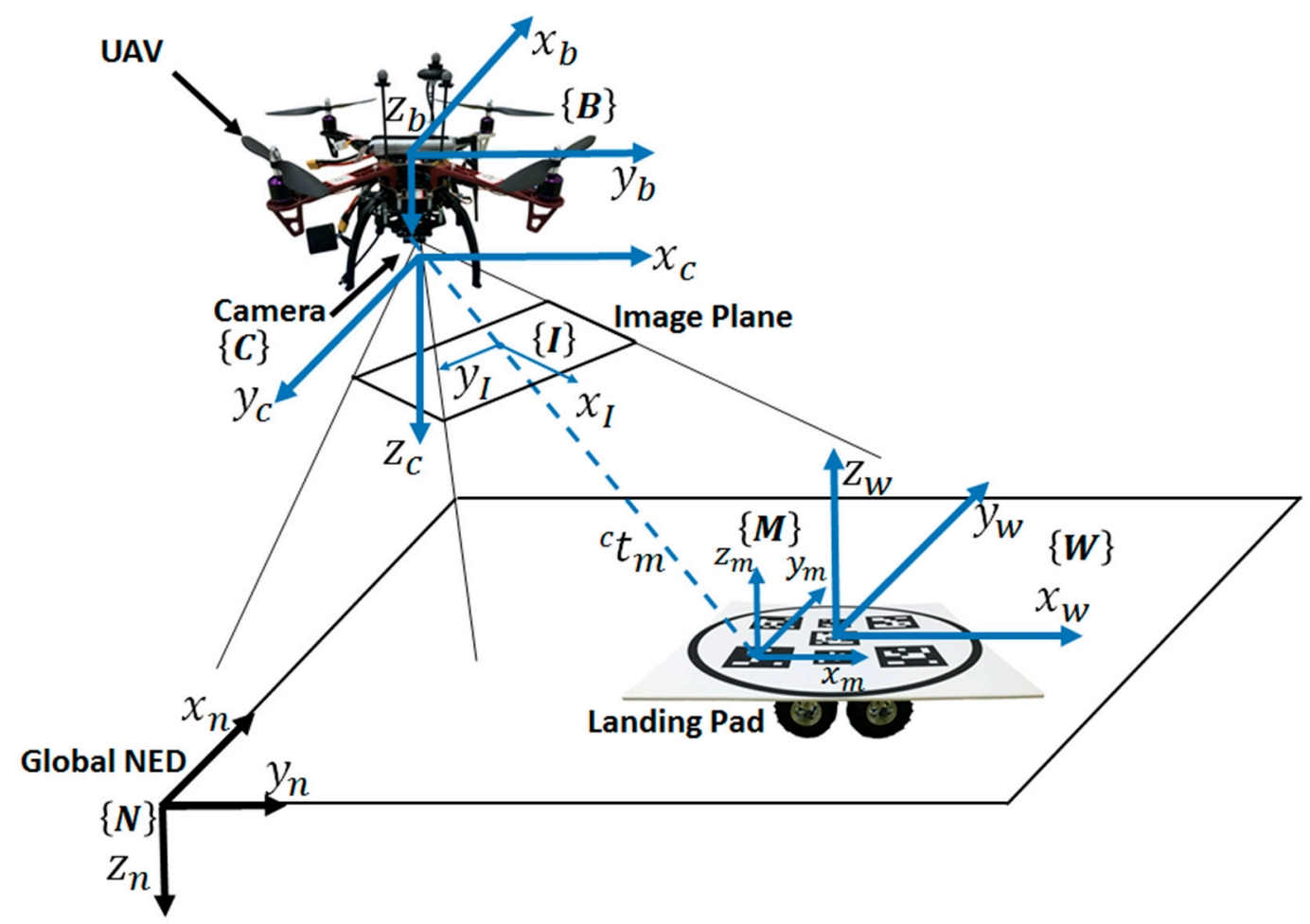

Figure 3. Definition of the unmanned aerial vehicle (UAV) body frame $B$, the camera frame $C$, the image frame $I$, the marker frame $M$, the world frame $W$, and a North-East-Down (NED) coordinate system taken as an inertial reference frame $N$. 
Let ${ }^{c} M_{m}$ be the homogeneous transformation matrix from the marker frame to the camera frame, which consists of a rotation matrix ${ }^{c} R_{m}(3 \times 3)$ and a translation vector ${ }^{c} t_{m}(3 \times 1)$. The coordinate transformation from frame $M$ to $C$ is given as:

$$
\left[\begin{array}{c}
x_{c} \\
y_{c} \\
z_{c} \\
1
\end{array}\right]={ }^{c} M_{m}\left[\begin{array}{c}
x_{m} \\
y_{m} \\
z_{m} \\
1
\end{array}\right]
$$

where $\left[x_{m}, y_{m}, z_{m}\right]^{T}$ is the position of the reference point in the marker frame, $\left[x_{c}, y_{c}, z_{c}\right]^{T}$ is the corresponding position in the camera frame, and transformation matrix ${ }^{c} M_{m}$ is:

$$
{ }^{c} M_{m}=\left[\begin{array}{cc}
{ }^{c} R_{m} & { }^{c} t_{m} \\
0^{T} & 1
\end{array}\right]
$$

In real flights, the camera frame rotates along with the quadrotor, while the $x_{m} y_{m}$ plane of the marker frame is kept horizontal. So, the inverse of ${ }^{c} M_{m}$ will be more useful in this paper, which is given as:

$$
{ }^{c} M_{m}={ }^{c} M_{m}^{-1}=\left[\begin{array}{cc}
{ }^{c} R_{m}^{-1} & -{ }^{c} t_{m}{ }^{c} R_{m}^{-1} \\
0^{T} & 1
\end{array}\right]
$$

where ${ }^{c} M_{m}$ is the transformation matrix from the camera frame to the marker frame, and the pose of the quadrotor with respect to marker i can be estimated with analysis of it. As the landing pad is a cooperative target and the origin of the world coordinates is set at the center of the landing pad, the pose of marker $i$ in the world frame is known. Thus, a translational transformation ${ }^{w} M_{m}$ from the marker frame to the world frame can be calculated, which is given as:

$$
\left[\begin{array}{c}
x_{w} \\
y_{w} \\
z_{w} \\
1
\end{array}\right]={ }^{w} M_{m}\left[\begin{array}{c}
x_{m} \\
y_{m} \\
z_{m} \\
1
\end{array}\right]
$$

In the captured image, any point can be positioned by pixel values, which is generally defined as $[u, v]^{T}$. With a calibrated camera, the reference point can be transformed from the pixel values to the camera coordinates, which is given as:

$$
\left[\begin{array}{l}
u \\
v \\
1
\end{array}\right]=M_{i n}\left[\begin{array}{c}
x_{n c} \\
y_{n c} \\
1
\end{array}\right]
$$

where $\left[x_{n c}, y_{n c}, 1\right]^{T}$ is the corresponding position on the normalized image plane, and $M_{i n}$ is referred to as the intrinsic parameter matrix of the camera, which is expressed as:

$$
M_{i n}=\left[\begin{array}{ccc}
f / d_{x} & 0 & u_{0} \\
0 & f / d_{y} & v_{0} \\
0 & 0 & 1
\end{array}\right]
$$

where $f$ is the focal length, $d_{x}$ and $d_{y}$ are the physical length per pixel in the $x$ and $y$ axis directions, respectively, $\left(u_{0}, v_{0}\right)$ is the intersection of the optical axis and the image plane in $2 \mathrm{D}$ pixel coordinates. As mentioned above, the landing pad and those planar patterns are located on the $z_{w}=0$ plane in $W$. Thus, the transformation from the normalized coordinates to the world coordinates can be expressed as: 


$$
\left[\begin{array}{c}
x_{n c} \\
y_{n c} \\
1
\end{array}\right]=H\left[\begin{array}{c}
x_{w} \\
y_{w} \\
1
\end{array}\right]
$$

where the $3 \times 3$ matrix $H$ is a planar homography defined as:

$$
H=\left[\begin{array}{lll}
h_{11} & h_{12} & h_{13} \\
h_{21} & h_{22} & h_{23} \\
h_{31} & h_{32} & h_{33}
\end{array}\right]
$$

where $h_{i j}$ is the i,j-th element of $H$, which leads to equations as follows:

$$
\left\{\begin{array}{l}
x_{n c}\left(h_{31} x_{w}+h_{32} y+h_{33}\right)=h_{11} x_{w}+h_{12} y_{w}+h_{13} \\
y_{n c}\left(h_{31} x_{w}+h_{32} y+h_{33}\right)=h_{21} x_{w}+h_{22} y_{w}+h_{23}
\end{array}\right.
$$

When an ArUco marker is decoded and recognized, its four corners are located in the pixel coordinates and their corresponding position in the world frame can be derived from the predefined identification and the actual size of the detected marker. Given at least four such correspondences, a set of linear equations can be established to solve the elements of $H$ :

$$
\left\{\begin{array}{ccccccccc}
x_{w}^{1} & y_{w}^{1} & 1 & 0 & 0 & 0 & -x_{n c}^{1} x_{w}^{1} & -x_{n c}^{1} y_{w}^{1} & -x_{n c}^{1} \\
0 & 0 & 0 & x_{w}^{1} & y_{w}^{1} & 1 & -y_{n c}^{1} x_{w}^{1} & -y_{n c}^{1} y_{w}^{1} & -y_{n c}^{1} \\
\vdots & \vdots & \vdots & \vdots & \vdots & \vdots & \vdots & \vdots & \vdots \\
x_{w}^{n} & y_{w}^{n} & 1 & 0 & 0 & 0 & -x_{n c}^{n} x_{w}^{n} & -x_{n c}^{n} y_{w}^{n} & -x_{n c}^{n} \\
0 & 0 & 0 & x_{w}^{n} & y_{w}^{n} & 1 & -y_{n c}^{n} x_{w}^{n} & -y_{n c}^{n} y_{w}^{n} & -y_{n c}^{n}
\end{array}\right\} h=0
$$

where $h$ is a $9 \times 1$ vector that contains all the $h_{i j}$ elements defined as $h=$ $\left[\begin{array}{lllllllll}h_{11} & h_{12} & h_{13} & h_{21} & h_{22} & h_{23} & h_{31} & h_{32} & h_{33}\end{array}\right]^{T},\left[\begin{array}{lll}x_{n c}^{i} & y_{n c}^{i} & 1\end{array}\right]^{T}$ is the $\mathrm{i}$-th reference point in the normalized coordinate, and $\left[\begin{array}{lll}x_{w}^{i} & y_{w}^{i} & 1\end{array}\right]^{T}$ is the homogeneous coordinate in the world frame. Therefore, the matrix equation is simplified as:

$$
A h=0
$$

Thus, the solution can be computed by using known methods such as singular value decomposition (SVD) and the average reprojection error $R_{a v g}^{E}$ can be minimized, which is defined as:

$$
R_{a v g}^{E}=\frac{\sum_{i=1}^{n}\left\|x_{n c}^{i}-H x_{w}^{i}\right\|}{n}
$$

where $n$ is the total number of corner correspondences.

\subsection{Fusion Estimation}

When more than one marker is recognized, pose estimates of different observed markers can be fused to form a better estimator. The previously estimated pose of the quadrotor with respect to the world frame given by every detected marker can be fused to get a more accurate result of the estimation. One way is to take a weighted average of the obtained estimates. Assuming that the estimator is unbiased, the variance of the original estimates should be taken into account to determine the weight coefficients. In general, the weights are chosen to minimize the variance of the weighted average such as a linear minimum variance estimator (LMVE). 
Let $p_{i}$ be the original pose estimate of $\mathrm{i}$-th detected marker, which is independent, and $\hat{p}$ be the unbiased estimate for the actual parameter $p$ after taking the weighted average, which is as follows:

$$
\hat{p}=\omega_{1} p_{1}+\omega_{2} p_{2}+\cdots \omega_{n} p_{n}
$$

where $\omega_{i}\left(\sum_{i=1}^{n} \omega_{i}=1\right.$ and $\omega_{i} \geq 0$ for all $\left.\mathrm{i}\right)$ is the weight that is to be determined, and $n$ is the total number of markers that are observed. The estimation error $\tilde{p}$ and known conditions are:

$$
\begin{aligned}
& \widetilde{p}=p-\hat{p} \\
& E(\widetilde{p})=0
\end{aligned}
$$

where $E(\widetilde{p})$ is the mathematical expectation; then, it can be proved by using the method of Lagrange multipliers that the variance is minimized when:

$$
\omega_{i}=\frac{\frac{1}{\operatorname{Var}\left(p_{i}\right)}}{\sum_{j=1}^{n} \frac{1}{\operatorname{Var}\left(p_{j}\right)}}
$$

and its minimum value $M S E_{\min }$ is:

$$
M S E_{\min }=\frac{1}{\sum_{i=1}^{n} \frac{1}{\operatorname{Var}\left(p_{i}\right)}}
$$

While the quadrotor starts to descend and approach the landing pad, the weight $\omega_{i}$ is updated in real time according to the total number of markers that are recognized and the noise of the original data, thus leading to a more accurate and reliable result.

\section{Dynamic Model and Landing System}

\subsection{Dynamic Model}

The quadrotors are gradually emerging as a popular platform in aerial robotics research due to their low cost, the ability to hover, and the simplicity in mechanical structure. The vehicle consists of four brushless motors and four propellers providing the necessary force and moment for 6DOF motion control. Unlike helicopters that need complex mechanical control linkages, the quadrotors rely on four individual motors and the variation in speed to control the vehicle, which greatly simplifies the whole system.

The 6DOF motion of a rigid quadrotor and corresponding frames are described in Figure 4.

The quadrotor vehicle is represented by a rigid body of mass $m$ and of moment of inertia $J$ along with external forces and torques caused by propellers and gravity. A local North-East-Down (NED) frame $N$ and a body fixed frame $B$ attached to the UAV at the center of mass are introduced to describe the motion of the quadrotor. Let ${ }^{n} p=\left[\begin{array}{lll}{ }^{n} p_{x} & { }^{n} p_{y} & { }^{n} p_{z}\end{array}\right]^{T}$ and ${ }^{n} v=\left[\begin{array}{lll}n_{v_{x}} & n_{v_{y}} & { }^{n} v_{z}\end{array}\right]^{T}$ be the position and linear velocity of the mass center relative to $N . \Theta=\left[\begin{array}{lll}\phi & \theta & \psi\end{array}\right]^{T}$ is defined as the roll/pitch/yaw angles, which describes the orientation of the quadrotor in $N$. The rotation matrix $R_{b}^{n}$ from $B$ to $N$ is given as:

$$
R_{b}^{n}=\left[\begin{array}{ccc}
\cos \theta \cos \psi & \sin \phi \sin \theta \cos \psi-\cos \phi \sin \psi & \cos \phi \sin \theta \cos \psi+\sin \phi \sin \psi \\
\cos \theta \sin \psi & \sin \phi \sin \theta \sin \psi+\cos \phi \cos \psi & \cos \phi \sin \theta \sin \psi-\sin \phi \cos \psi \\
-\sin \theta & \sin \phi \cos \theta & \cos \phi \cos \theta
\end{array}\right]
$$




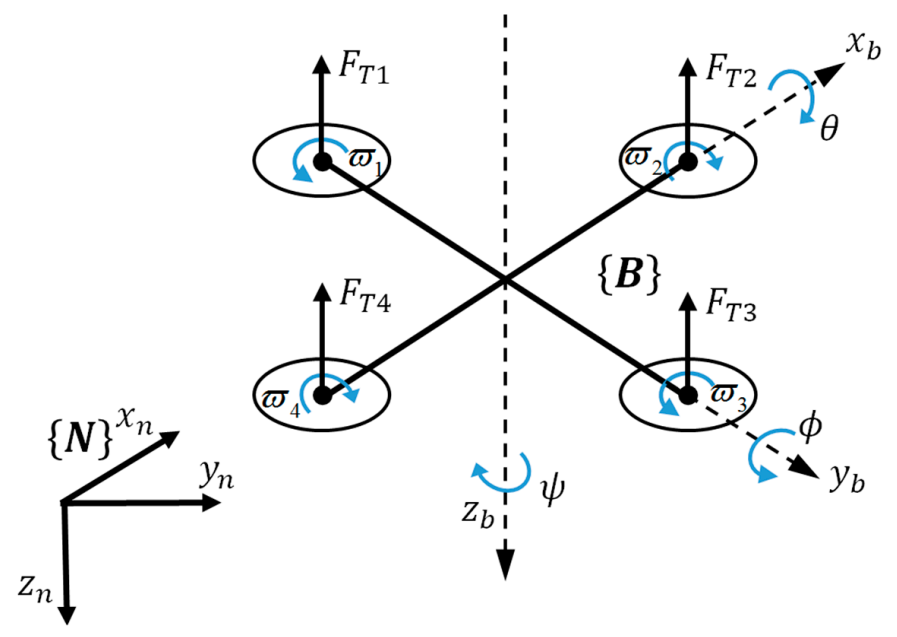

Figure 4. 6DOF motion of a rigid quadrotor with corresponding frames.

The equations of motion for the quadrotor can be described as:

$$
\begin{gathered}
n_{\dot{p}}={ }^{n_{v}} \\
n_{\dot{v}}=g n_{3}-\frac{f_{b}}{m} R_{b}^{n} n_{3} \\
J \cdot{ }^{b} \dot{\omega}=-{ }^{b} \omega \times\left(J \cdot{ }^{b} \omega\right)+G_{a}+\tau
\end{gathered}
$$

where $f_{b}$ is the translational force applied to the quadrotor expressed in $B, \tau$ is the torque, $g$ is the acceleration due to gravity, and ${ }^{b} \omega$ is the angular velocity of the MAV in frame $B$. The gyroscopic moment $G_{a}$, which is mainly generated by propellers, is not considered in this case. Furthermore, the translational dynamics shown in Equations (19) and (20) can be simplified as:

$$
\begin{aligned}
& n_{\ddot{p}_{x}}=-\frac{f_{b}}{m}(\sin \phi \sin \psi+\cos \phi \sin \theta \cos \psi) \\
& n \ddot{p}_{y}=-\frac{f_{b}}{m}(-\sin \phi \cos \psi+\cos \phi \sin \theta \sin \psi) \\
& n_{\ddot{p}_{z}}=g-\frac{f_{b}}{m} \cos \phi \cos \theta
\end{aligned}
$$

Furthermore, it can be assumed that $\sin \phi \approx 1, \cos \phi \approx 1, \sin \theta \approx \theta$, and $\cos \theta \approx 1$, considering that the roll and pitch angles are very small, which leads to a simplified dynamic model as described in [38].

\subsection{Flight Control Algorithm}

Since the quadrotor is an underactuated system with four independent inputs, only the desired position $\left[\begin{array}{lll}x_{d} & y_{d} & z_{d}\end{array}\right]^{T}$ and desired attitude $\psi_{d}$ can be directly tracked. Other variables such as $\phi_{d}$ and $\theta_{d}$ are determined by the known ones. The hierarchical control scheme of a quadrotor system is shown in Figure 5.

The position $\left[\begin{array}{lll}x_{i} & y_{i} & z_{i}\end{array}\right]^{T}$ and yaw angle $\psi_{i}$ of the quadrotor from marker $i$ are estimated by the onboard vision system in the world frame, while the roll and pitch angles given by the onboard microcontroller using an EKF are expressed in global NED. The state of the quadrotor, the control desired position $\left[\begin{array}{lll}x_{d} & y_{d} & z_{d}\end{array}\right]^{T}$, and the yaw angle $\psi_{d}$ are all expressed in the global NED. The position of the landing pad in global NED is exploited to calculate the state of the quadrotor. Cascade PID controllers are designed to individually control the 3D position and yaw angle of the quadrotor. The inner-loop 
controller for attitude control has been implemented on an open source autopilot. All PID gains have been preliminarily tuned in hovering flight tests. Considering that the thrust value is determined not only by the input of the desired position but also by the total takeoff weight of the quadrotor, the height controller is divided into two parts: a slightly changed base value for hovering and a fast controller for position control. An overview of the proposed landing system including landing pad recognition, $6 \mathrm{DOF}$ pose estimation, and flight control is shown in Figure 6.

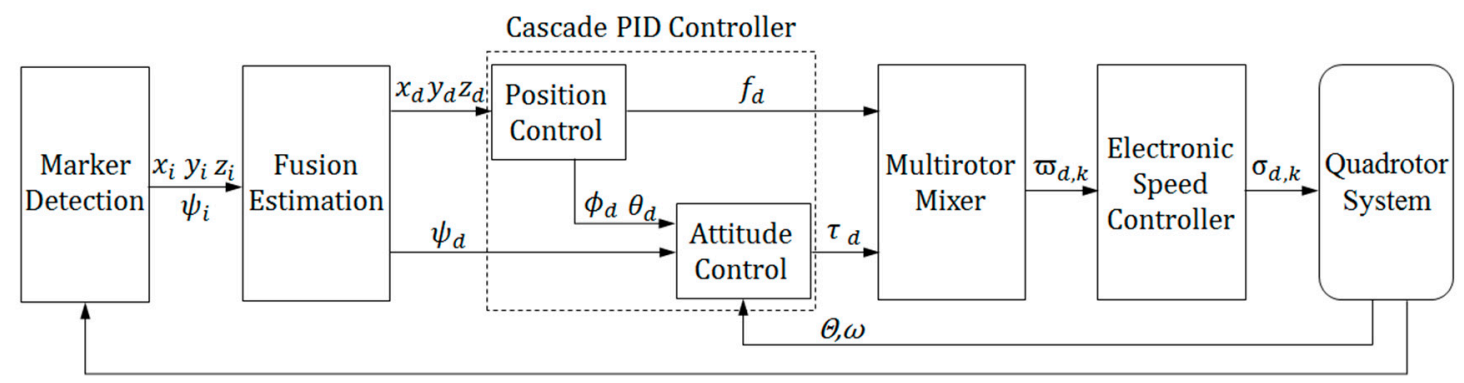

Figure 5. Hierarchical control architecture of a quadrotor.

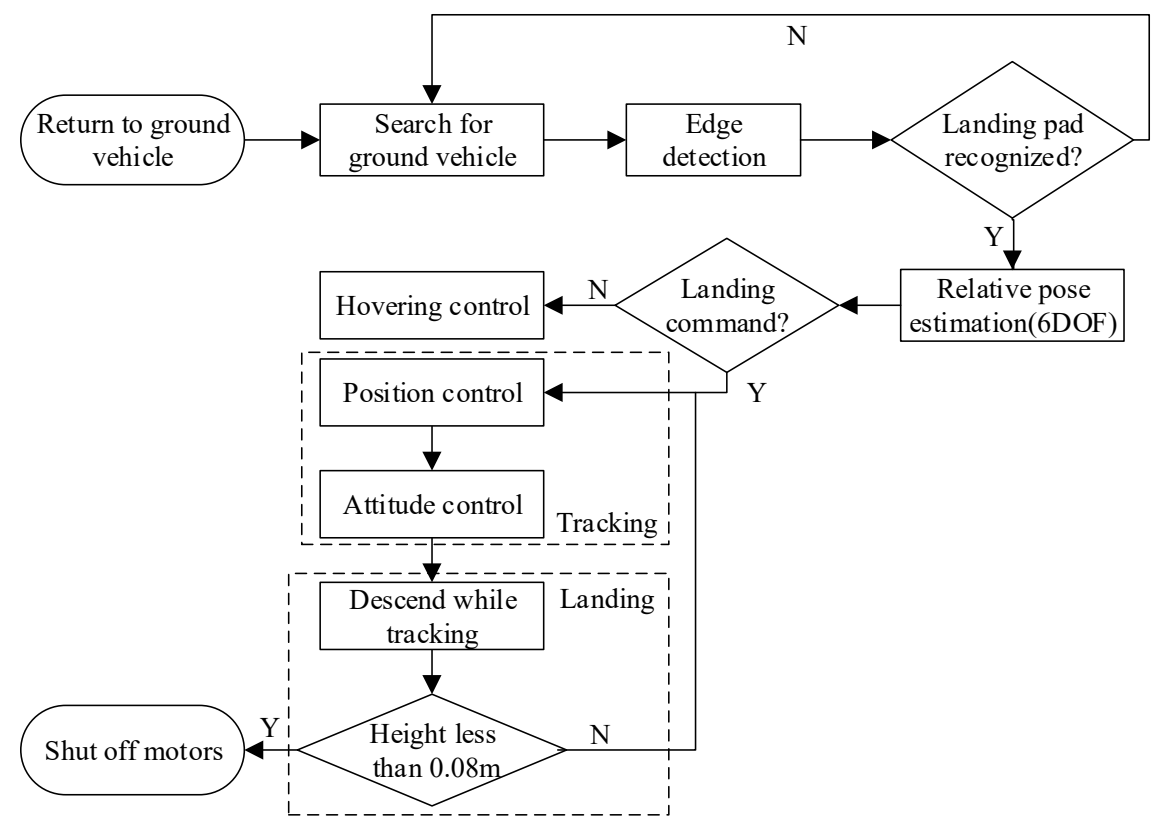

Figure 6. Overview of the proposed autonomous landing system.

When the landing pad is recognized by the onboard vision system, the quadrotor will maintain a constant descending velocity while continuing to track the target. With the landing pad mentioned above, the estimates obtained by the proposed system will not be divergent when the quadrotor is close to the target. Finally, due to the ground effect on the quadrotor, the motors are programmed to be shut off directly when the height is under $0.08 \mathrm{~m}$ and the horizontal distance must be less than $0.1 \mathrm{~m}$ simultaneously.

\section{Experiments and Results}

\subsection{Experimental Setup}

Most of the experimental UAVs are rather expensive with high-precision sensors and devices, which are unaffordable for practical applications. Instead of depending on high-resolution cameras that may cost more than $\$ 400$, a low-cost consumer camera is used to establish the onboard vision system. The camera at a dimension of $35 \times 35 \times 30 \mathrm{~mm}^{3}$ weighs only 50 grams and has a maximum resolution 
of $1280 \times 720$ at 30 frames per second (fps), which makes it an ideal onboard sensor (see Figure 7a). With a constant focus length of $3.6 \mathrm{~mm}$, the camera is aimed downward and attached to the bottom of the fuselage, covering a diagonal FOV of $90^{\circ}$. The price of only $\$ 10$ is very attractive, considering its unexpected great performance. After calibration, the intrinsic parameters of the onboard camera are $f / d_{x}=912.5796, f / d_{y}=909.4341, u_{0}=669.2593$ and $v_{0}=322.0985$, while the distortion coefficient vector is $\left[\begin{array}{lllll}0.0486 & -0.0907 & 0.0003 & -0.0009 & 0\end{array}\right]^{T}$.

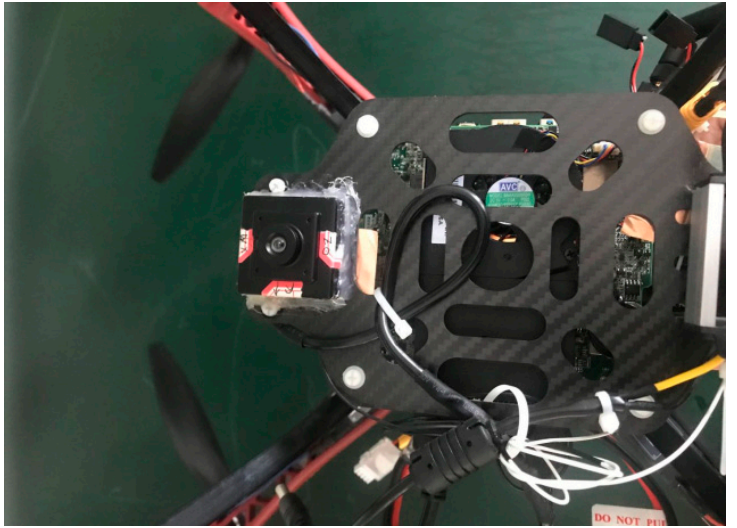

(a)

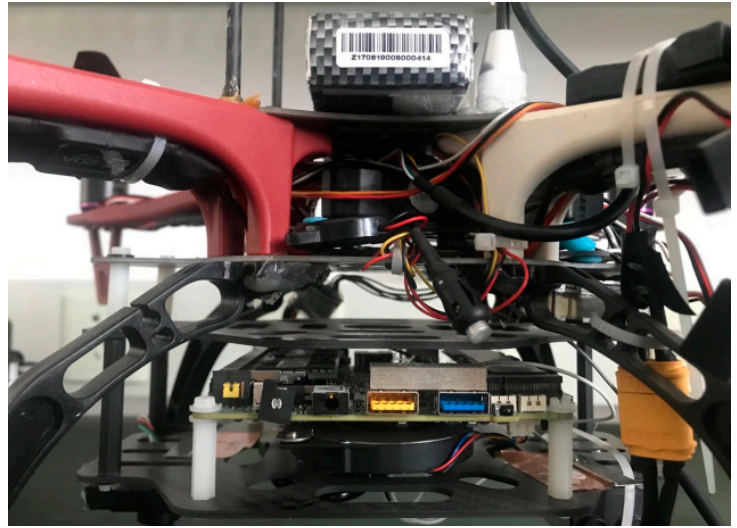

(b)

Figure 7. The low-cost camera (a) and onboard computer (b).

As shown in Figure 8, the self-customized quadrotor is $45 \mathrm{~cm}$ in diameter based on the DJI F450 frame, and weighs 1.6 kilograms, including all the onboard devices and payloads. A $3300 \mathrm{mAh}$ lithium battery powers all the motors and onboard electronics, which leads to a maximum flight time of up to $25 \mathrm{~min}$.

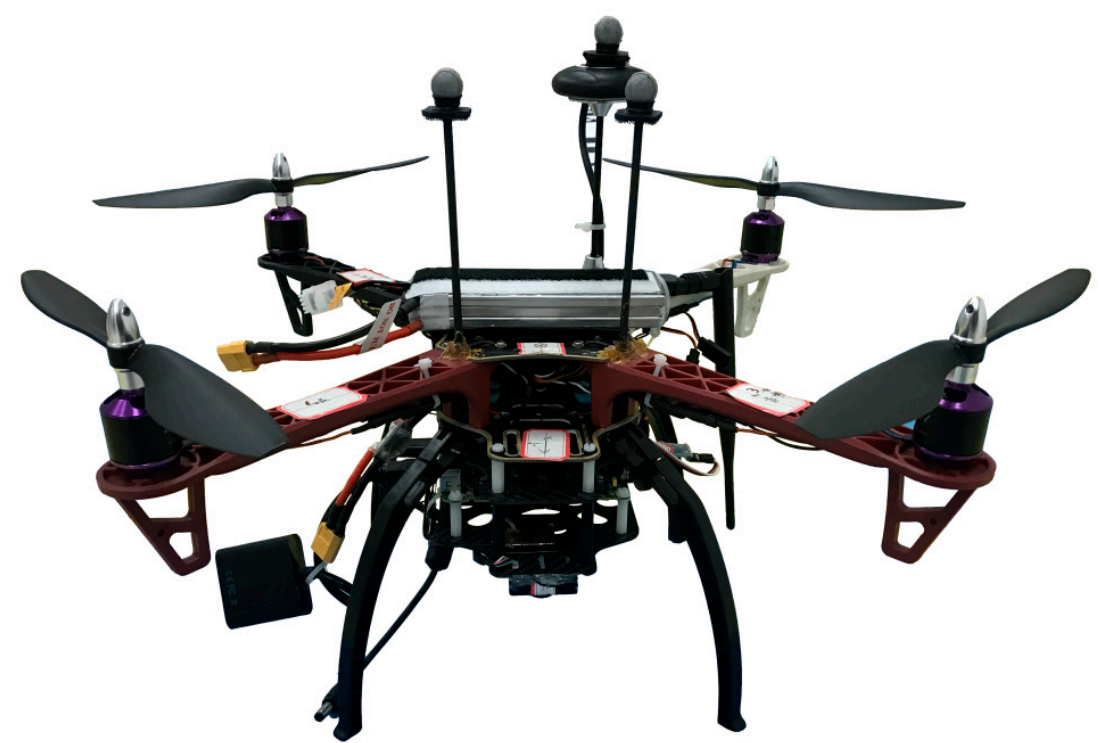

Figure 8. Overlook of the experimental platform in this paper.

The overall system setup to perform the flight experiments for validation of the landing control algorithm is shown in Figure 9. 


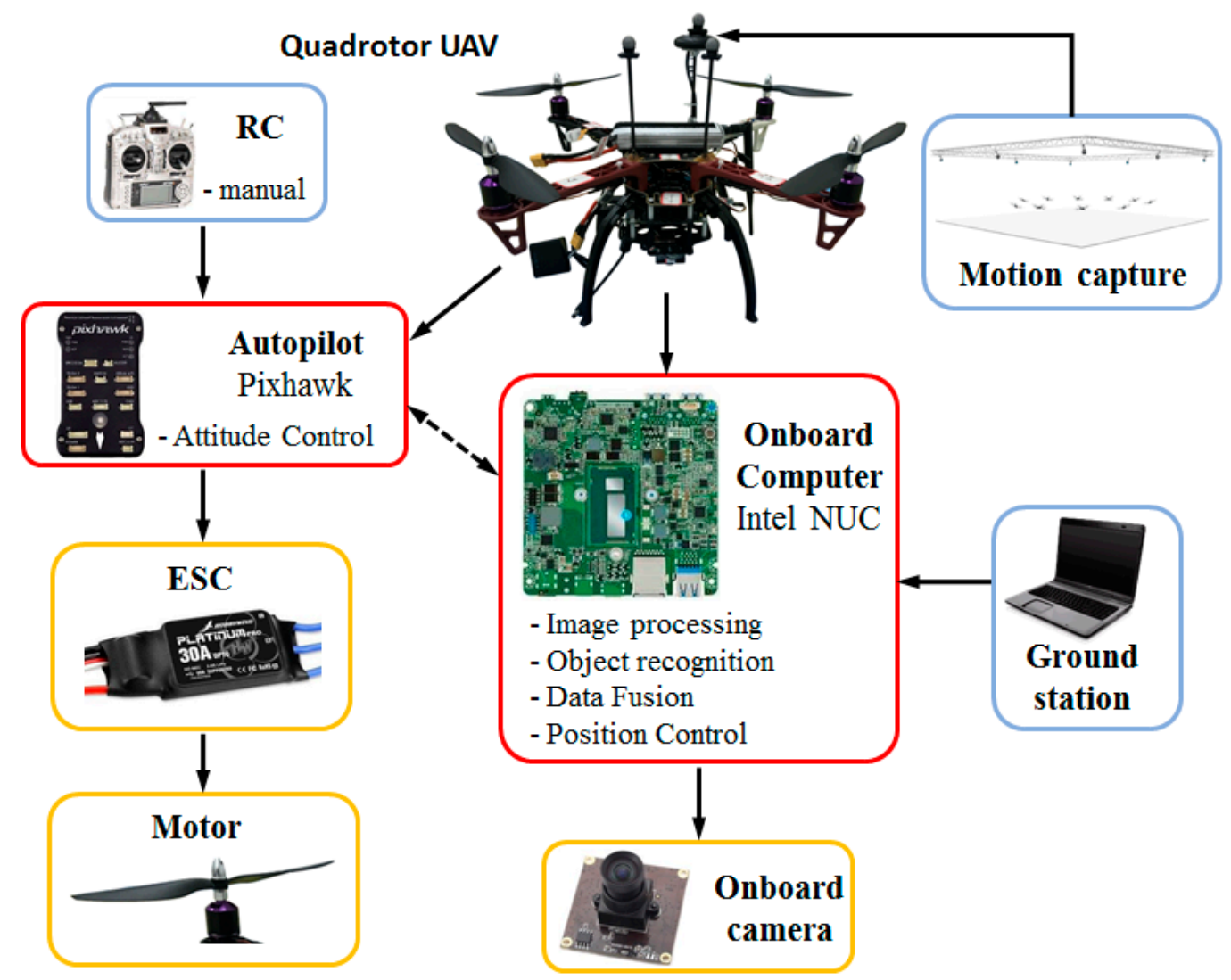

Figure 9. The architecture of the experimental system.

The inner-loop stabilization and attitude control of the quadrotor is achieved by utilizing a microcontroller developed by the Pixhawk team at ETU Zürich [39]. The Pixhawk controller is equipped with dual inertial measurement units (IMU) in case any one of them is malfunctioning. Many additional sensors and devices can be supported via drivers released by developer communities. The firmware version selected in this paper is 1.5.5.

In order to achieve more complex operations such as image processing, object recognition, data fusion, and position control, an Intel NUC with Core i5 processor is used as an onboard computer to perform the proposed vision algorithm. Based on serial communication, the current status and pose estimates can be transferred from the NUC to the Pixhawk flight controller as input information, which is developed based on a MAVLINK [40] extendable communication node for the Robot Operation System (ROS) [41], as shown in Figure 10. A higher estimation and control rate will significantly improve the accuracy, if supported by the hardware. However, in this paper, the estimation rate is at $25 \mathrm{~Hz}$, and is limited by the onboard computation power. Thus, the control rate cannot be faster than $25 \mathrm{~Hz}$ under the current conditions. By using a $2.4 \mathrm{GHz}$ remote controller (RC), the quadrotor can be controlled manually at the beginning of the experiment, and then switched to the offboard mode triggered by an RC signal. 


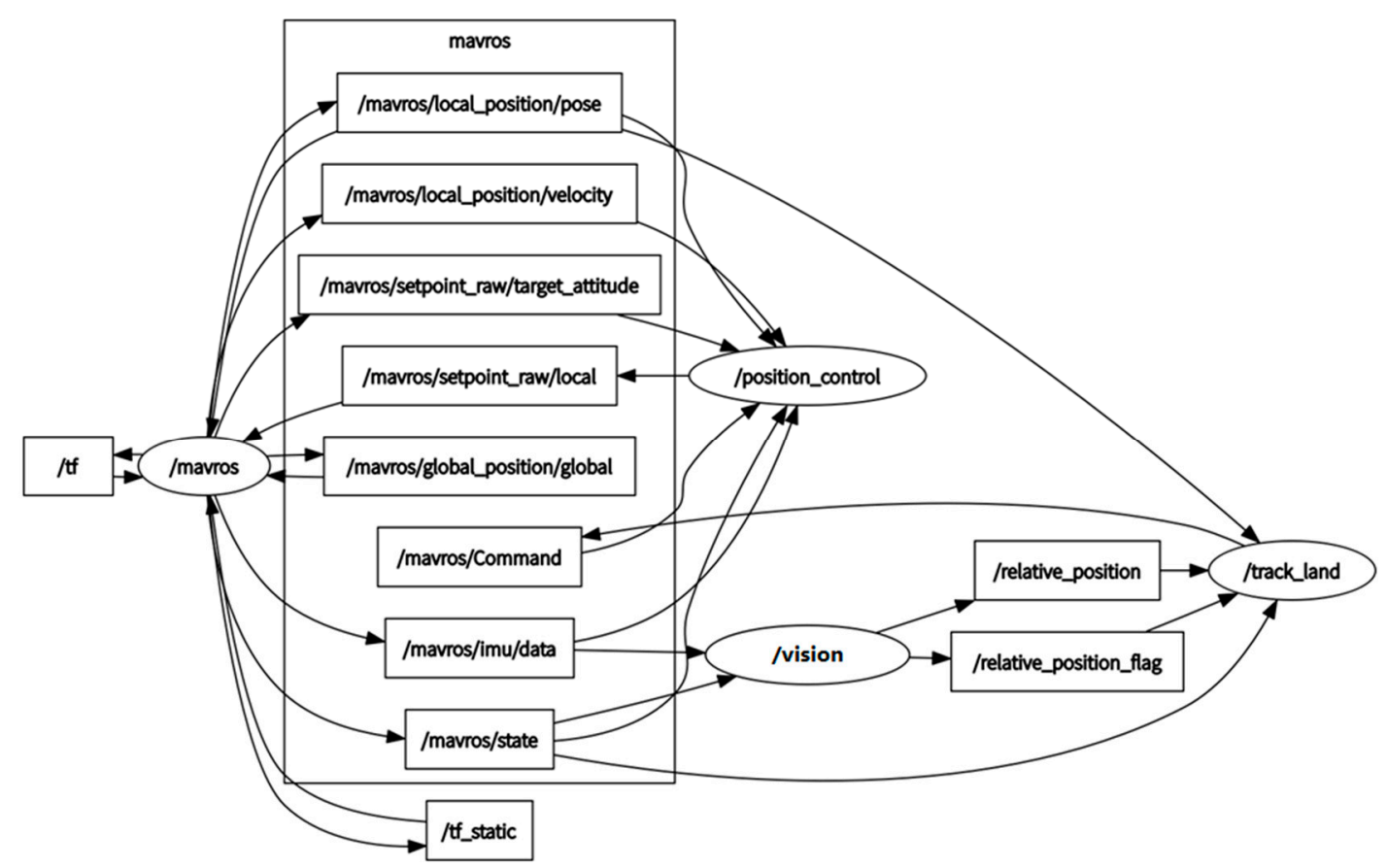

Figure 10. Robot Operation System (ROS) nodes and topics created to implement the algorithm.

ROS nodes are basic executable programs that process information to communicate with other nodes. In this system, the necessary nodes and topics shown in Figure 10 have been created and used to achieve the vision-based autonomous landing algorithm. Predefined topics can be published or subscribed by these nodes, thus passing the messages from one to another.

Once the landing procedure is launched, the quadrotor is in a fully autonomous mode, and only the onboard images along with IMU data can be utilized to provide navigation information. Since no GPS data can be received in indoor environments, a motion capture system (OptiTrack [42]) is used to provide the $6 \mathrm{DOF}$ pose of the quadrotor in real time at $100 \mathrm{~Hz}$ as the ground truth data, which can be compared with the vision system. To evaluate the proposed system, pose estimates of each detected marker have also been recorded as a reference value.

An autonomous landing test is shown in Figure 11.

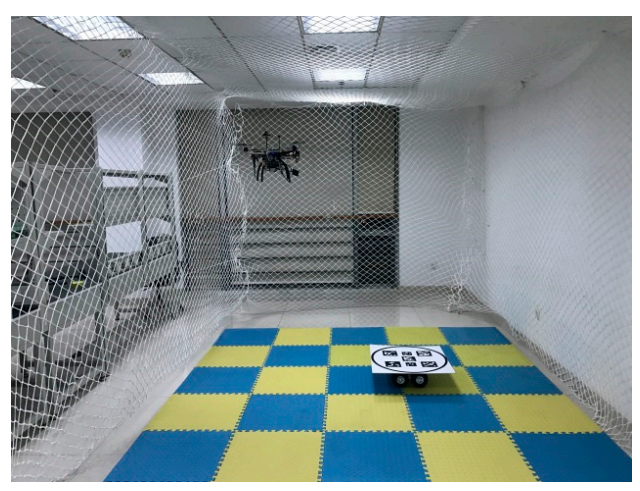

(a)

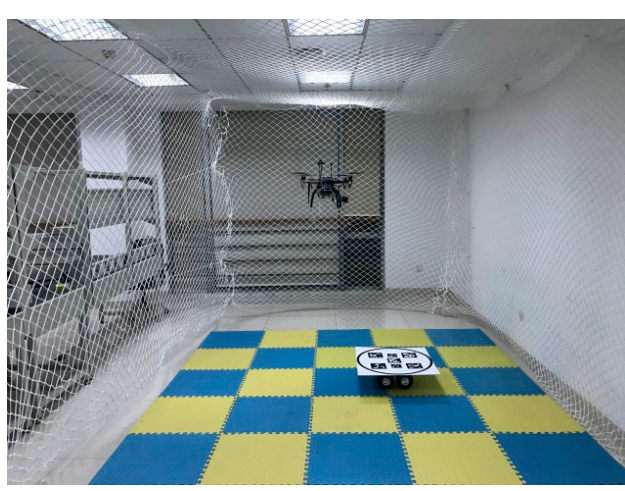

(b)

Figure 11. Cont. 


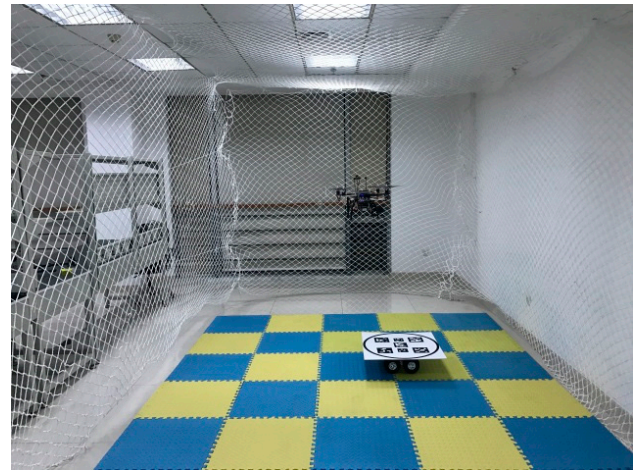

(c)

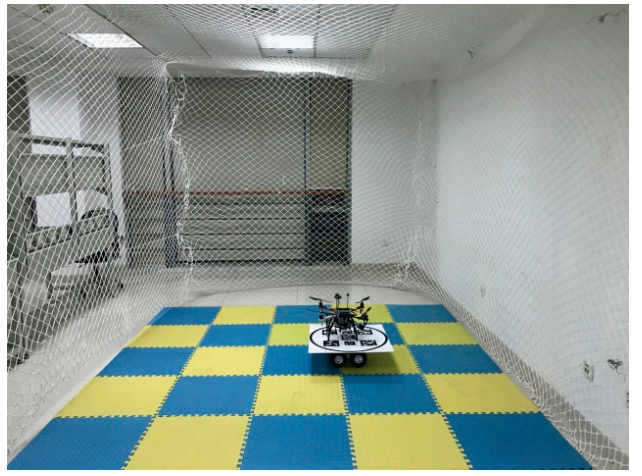

(d)

Figure 11. Autonomously approaching $(\mathbf{a}, \mathbf{b})$, descending (c), and landing on a static landing pad (d).

\subsection{Hovering Flight Control and Accuracy Analysis}

During the indoor experiments, the GPS and air pressure sensor for position control were disabled. The desired yaw angle was set to be zero degrees. Under such conditions, a series of hovering and hand-held tests at different heights have been conducted with the onboard vision system. Compared with hand-held cases, the vibration of the quadrotor in real flight could increase errors to the pose estimates, but the actual impact was not so great in hovering flight. This is not a surprise, because the roll and pitch angles are quite small, which will not introduce large deviations. By comparing to the ground truth data, the root mean square errors (RMSEs) of the proposed vision algorithm during the whole flight are listed in Table 1 (in the Estimated row). As a contrast, the data without fusion estimation is listed (in the Single marker row).

Table 1. Root mean square errors (RMSEs) of hovering flight at $1 \mathrm{~m}$ in different cases.

\begin{tabular}{cccccc}
\hline RMSEs $(\mathbf{m m})$ & $\mathbf{x}$ & $\mathbf{y}$ & $\mathbf{z}$ & xy Plane & 3D \\
\hline Single marker & 30.8 & 44.0 & 8.9 & 53.7 & 54.4 \\
Estimated & 10.1 & 19.1 & 5.5 & 21.6 & 22.2 \\
\hline
\end{tabular}

In about $82 \mathrm{~s}$ flight at $1 \mathrm{~m}$ height, an accuracy of $\pm 10.1 \mathrm{~mm}$ in the $\mathrm{x}$ position and $\pm 19.1 \mathrm{~mm}$ in the $\mathrm{y}$ position could be achieved, which is much better than that of a single ArUco marker. The deviation of 3D position estimates is $22.2 \mathrm{~mm}$, thus leading to an accuracy of $23.2 \mathrm{~mm}$ in hovering control measured by the motion capture system. Figure 12 shows a more detailed record of the flight. Results of the proposed vision system are plotted in blue, the single marker is plotted in green, and the ground truth data are plotted in red.

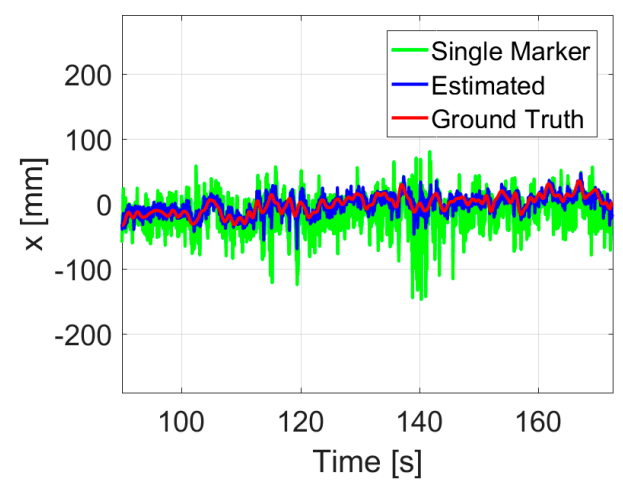

(a)

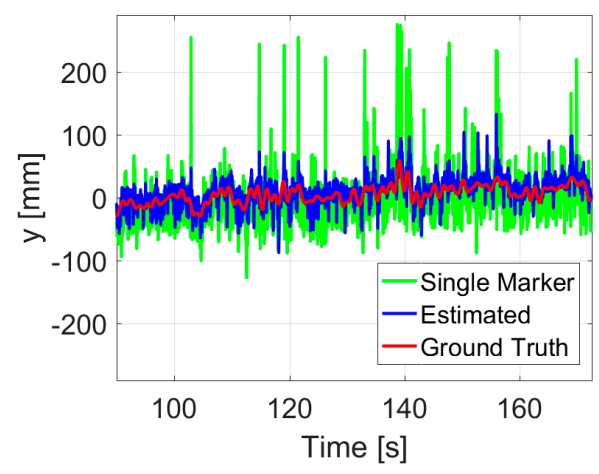

(b)

Figure 12. Cont. 


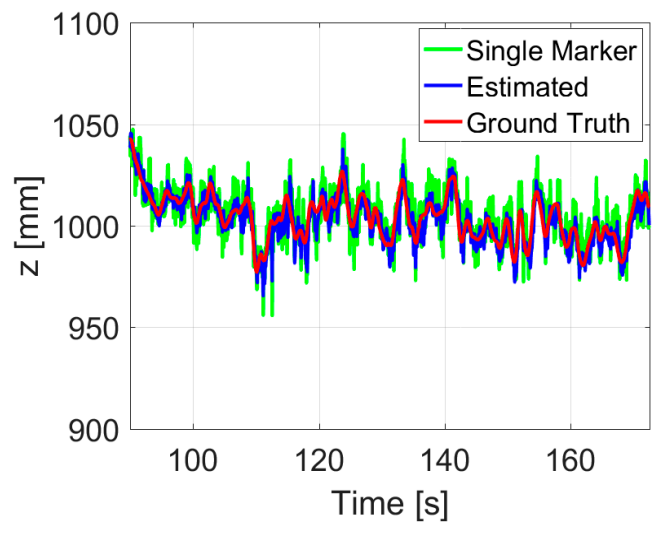

(c)

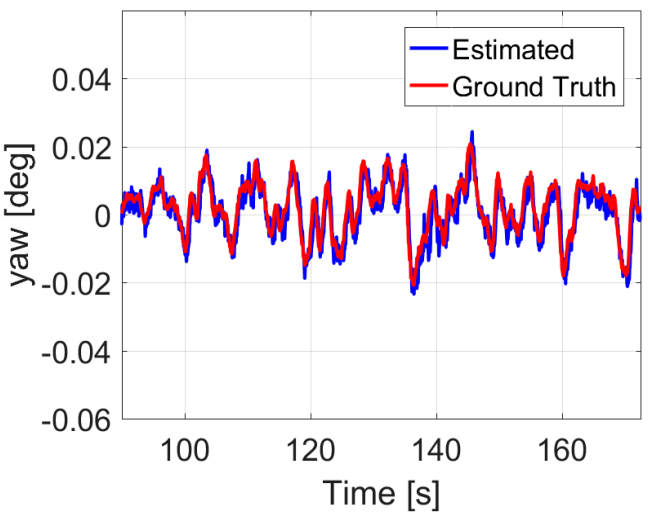

(d)

Figure 12. Position $(\mathbf{a}-\mathbf{c})$ and yaw angle (d) estimates during a hovering flight at $1 \mathrm{~m}$ height.

It is obvious that the proposed estimates plotted in blue are well in line with the ground truth data throughout the flight test. Outliers caused by image noises have been significantly filtered by the proposed fusion estimation method. With the low-cost onboard vision system, high-precision control of autonomous hovering flight is achieved.

\subsection{Autonomous Landing}

This section presents the results of autonomous landing on the static landing pad, which is fixed on a ground platform. Once the quadrotor is armed and switched to the offboard mode, it will take off autonomously to the set point and keep hovering until the next command is received. As shown in Figure 13, after 7 seconds of hovering around the set point $(0,0,1900)^{T}(\mathrm{~mm})$, the quadrotor received a command to land, and it took about $8 \mathrm{~s}$ to descend. Then, the onboard system started to determine whether the shut-off condition was met or not. The process was repeated until the destination area was reached, where the quadrotor could be blindly powering down. The final position of the quadrotor is very close to the center of the landing pad, which reflects the significance of this novel landing pad. The results are plotted in the world frame to better show the relative pose of the quadrotor with respect to the landing pad.

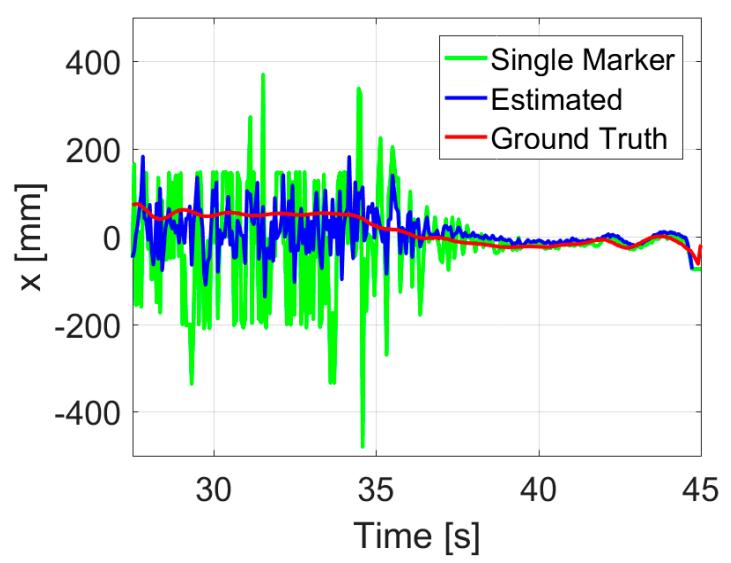

(a)

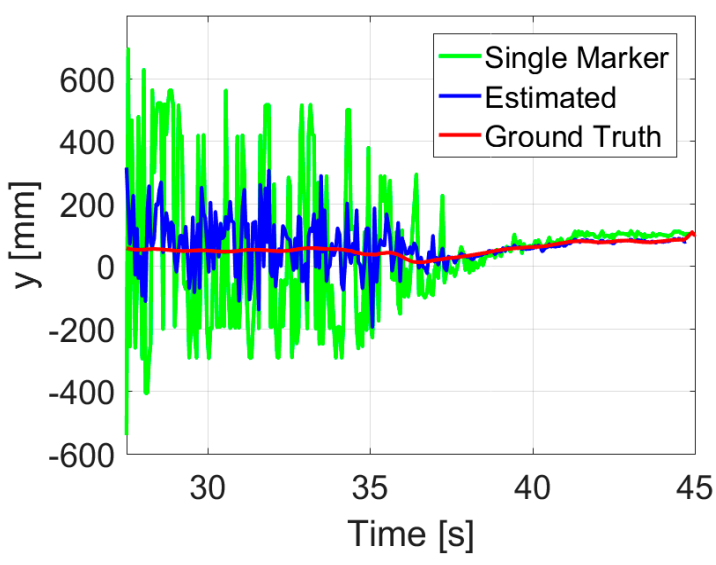

(b)

Figure 13. Cont. 


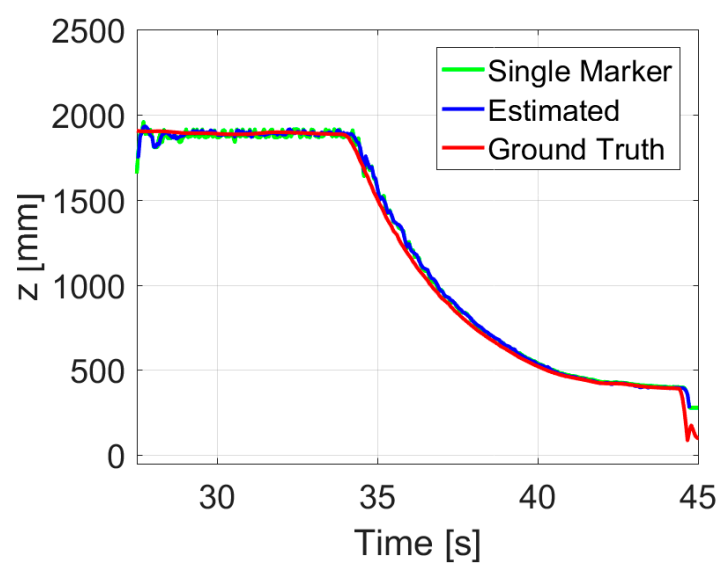

(c)

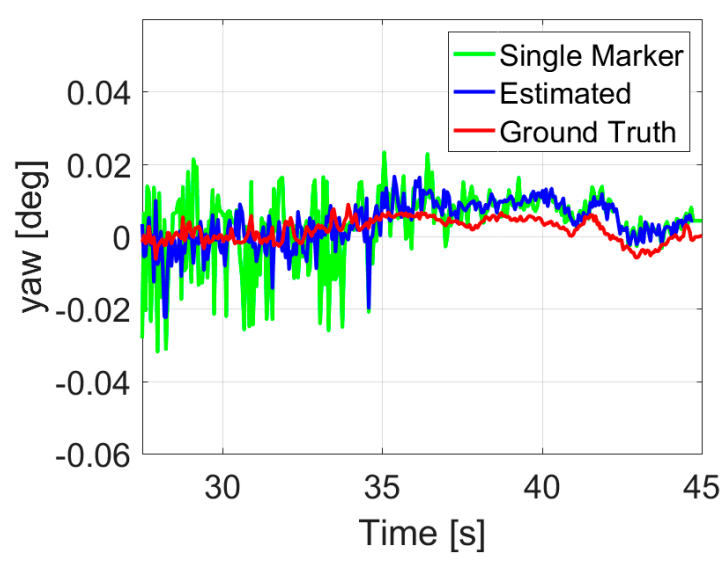

(d)

Figure 13. Position (a-c) and yaw angle (d) estimates during an autonomous landing.

Figure 14 shows the pose estimates in a successful takeoff, hovering, moving, and landing flight. For the beginning of the takeoff phase, the quadrotor ascended without closed-loop control until one of the markers was recognized. A command made by the ground station was sent to the onboard system, and then the quadrotor moved to the set point $(550,-550,2100)^{T}(\mathrm{~mm})$. After hovering about $10 \mathrm{~s}$, it started the landing phase, and did not take much time to meet the shut-off condition.

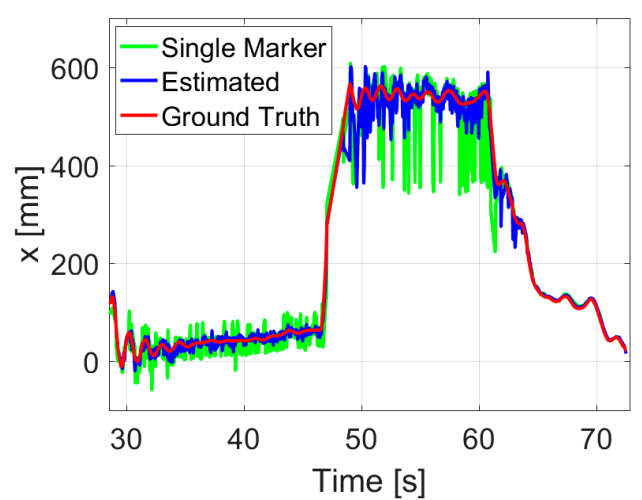

(a)

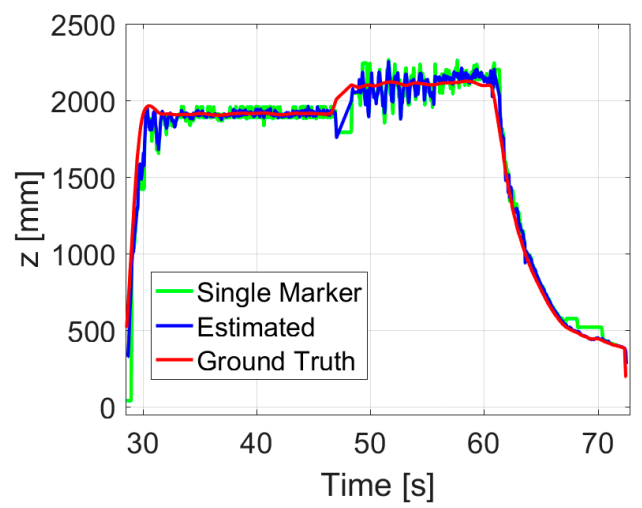

(c)

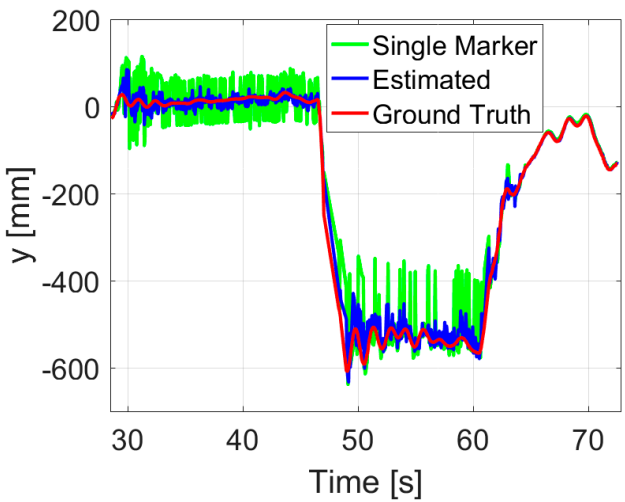

(b)

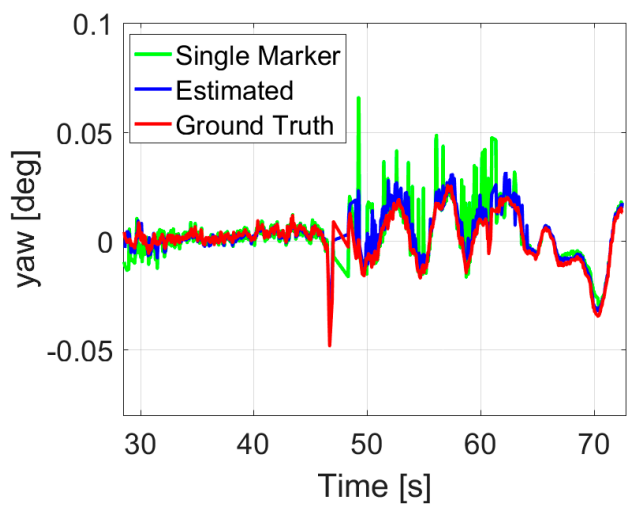

(d)

Figure 14. Position $(\mathbf{a}-\mathbf{c})$ and yaw angle (d) estimates during an indoor autonomous takeoff, hovering, and landing flight. 
Some oscillations still remain in the current configuration, which occurred with sudden changes of the quadrotor's attitude. In this paper, the camera is fixed to the bottom of the quadrotor, and so it rotates along with the body frame. The estimation error will significantly increase when the orientation of the camera changes quickly in a very short time, especially at a relatively low estimation rate. A higher operating frequency would significantly improve the stabilization, but is prevented by the limited ability of onboard image processing. In fact, considering the cost of all the sensors and devices, the performance of the proposed vision system is quite attractive in practical applications.

A video of these experiments demonstrating autonomous flights with the proposed system is available at Supplementary Materials section.

\section{Conclusions}

In this paper, we have presented an onboard vision system that consists of a downward-looking camera, a microcontroller, and an image processor to visually and autonomously provide position and attitude estimates of the MAV with respect to the ground platform. A novel landing pad using different ArUco marker sizes is carefully designed for autonomous landing tasks, ensuring the detectability at different distances. With the proposed algorithm, position and attitude estimates of the MAV can be calculated by utilizing reference points extracted from the pad image. A method of fusion estimation has proved its effectiveness in experiments.

We demonstrated the proposed vision algorithm through a series of experiments, which enabled a self-customized quadrotor to autonomously take off from, hover above, track, and land on a static landing pad. Evaluated by an external tracking system, the results of real-time flight experiments have shown the feasibility, robustness, and accuracy of the vision algorithm. Considering that the cost of sensors is very low, the achieved accuracy is very attractive and sufficient for autonomous landing tasks.

There are several directions in which future work is of interest. To achieve more robust and accurate measurements, the sensor fusion of both IMU data and the vision system is worth investigating. Extra sensors such as infrared camera and Lidar may be used to expand the application of MAVs. Furthermore, we plan to place the camera in a gimbaled platform, which enables the MAV to track and approach a moving target.

Supplementary Materials: The following are available online at http://www.mdpi.com/1424-8220/19/21/ 4703/s1, Video S1: Onboard Monocular Vision System for Autonomous Landing of a Low-Cost MAV in GPS-Denied Environment.

Author Contributions: Conceptualization, X.L. and S.Z.; Methodology, X.L.; Software, X.L.; Data curation, J.T.; formal analysis, J.T.; investigation, X.L.; funding acquisition, L.L.; validation, S.Z.

Funding: This research was funded by the National Natural Science Foundation of China under Grant 11802334.

Acknowledgments: The authors would like to thank Rongwei Li and Qi Xiao for their contribution on the practical experiment configuration.

Conflicts of Interest: The authors declare no conflict of interest.

\section{References}

1. Sun, J.; Li, B.; Jiang, Y.; Wen, C. A Camera-Based Target Detection and Positioning UAV System for Search and Rescue (SAR) Purposes. Sensors 2016, 16, 1778. [CrossRef] [PubMed]

2. Koppány, M.; Lucian, B. Vision and control for uavs: A survey of general methods and of inexpensive platforms for infrastructure inspection. Sensors 2015, 15, 14887-14916.

3. Jung, Y.; Lee, D.; Bang, H. Study on Ellipse Fitting Problem for Vision-based Autonomous Landing of an UAV. In Proceedings of the 14th International Conference on Control, Automation and Systems (ICCAS), Seoul, Korea, 22-25 October 2014; pp. 1631-1634.

4. Saripalli, S.; Montgomery, J.F.; Sukhatme, G.S. Visually Guided Landing of an Unmanned Aerial Vehicle. IEEE Trans. Robot. Autom. 2003, 19, 371-380. [CrossRef] 
5. Vetrella, A.R.; Fasano, G.; Accardo, D.; Moccia, A. Differential GNSS and Vision-Based Tracking to Improve Navigation Performance in Cooperative Multi-UAV Systems. Sensors 2016, 16, 2164. [CrossRef]

6. Wenzel, K.E.; Rosset, P.; Zell, A. Low-cost visual tracking of a landing place and hovering flight control with a microcontroller. J. Intell. Robot. Syst. 2010, 57, 297-311. [CrossRef]

7. Yang, S.; Scherer, S.A.; Zell, A. An onboard monocular vision system for autonomous takeoff, hovering and landing of a micro aerial vehicle. J. Intell. Robot. Syst. 2013, 69, 499-515. [CrossRef]

8. Chen, Z.; Huang, J.B. A vision-based method for the circle pose determination with a direct geometric interpretation. IEEE Trans. Robot. Autom. 1999, 15, 1135-1140. [CrossRef]

9. Forsyth, D.; Mundy, J.L.; Zisserman, A.; Coelho, C.; Heller, A.; Rothwell, C. Invariant descriptors for 3d object recognition and pose. IEEE Trans. Pattern Anal. Mach. Intell. 1991, 13, 971-991. [CrossRef]

10. He, L.; Chao, Y.; Suzuki, K. A run-based two-scan labeling algorithm. IEEE Trans. Image Process. 2008, 17, 749-756.

11. Sanchez-Lopez, J.L.; Pestana, J.; Saripalli, S.; Campoy, P. An Approach Toward Visual Autonomous Ship Board. J. Intell. Robot. Syst. 2014, 74, 113-127. [CrossRef]

12. Srinivasan, M.V.; Zhang, S.W.; Chahl, J.S.; Barth, E.; Venkatesh, S. How honeybees make grazing landings on flat surfaces. Biol. Cybern. 2000, 83, 171-183. [CrossRef] [PubMed]

13. Koenderink, J.J.; Van, A.D. Facts on optic flow. Biol. Cybern. 1987, 56, 247-254. [CrossRef] [PubMed]

14. Herisse, B.; Hamel, T.; Mahony, R.; Russotto, F.X. Landing a VTOL Unmanned Aerial Vehicle on a Moving Platform Using Optical Flow. IEEE Trans. Robot. 2012, 28, 77-89. [CrossRef]

15. Yang, S.; Scherer, S.A.; Schauwecker, K.; Zell, A. Autonomous Landing of MAVs on an Arbitrarily Textured Landing Site Using Onboard Monocular Vision. J. Intell. Robot. Syst. 2014, 74, 27-43. [CrossRef]

16. Chaves, S.M.; Wolcott, R.W.; Eustice, R.M. Neec Research: Toward GPS-Denied Landing of Unmanned Aerial Vehicles on Ships at Sea. Nav. Eng. J. 2015, 127, 23-35.

17. Olson, E. AprilTag: A robust and flexible visual fiducial system. In Proceedings of the IEEE International Conference on Robotics and Automation (ICRA), Shanghai, China, 9-13 May 2011; pp. 3400-3407.

18. Lee, H.; Jung, S.; Shim, D.H. Vision-based UAV landing on the moving vehicle. In Proceedings of the International Conference on Unmanned Aircraft Systems (ICUAS), Arlington, VA, USA, 8-10 June 2016.

19. Huang, L.; Song, J.; Zhang, C. Observability Analysis and Filter Design for a Vision Inertial Absolute Navigation System for UAV Using Landmarks. Optik 2017, 149, 455-468. [CrossRef]

20. Tong, Q.; Peiliang, L.; Shaojie, S. Vins-mono: A robust and versatile monocular visual-inertial state estimator. IEEE Trans. Robot. 2018, 34, 1004-1020.

21. Shen, S.; Mulgaonkar, Y.; Michael, N.; Kumar, V. Vision-based state estimation for autonomous rotorcraft MAVs in complex environments. In Proceedings of the IEEE International Conference on Robotics and Automation (ICRA), Karlsruhe, Germany, 6-10 May 2013.

22. Sanchez-Lopez, J.L.; Arellano-Quintana, V.; Tognon, M.; Campoy, P.; Franchi, A. Visual Marker based Multi-Sensor Fusion State Estimation. In Proceedings of the 20th IFAC World Congress, Toulouse, France, 9-14 July 2017.

23. Rekimoto, J. Matrix: A Realtime Object Identification and Registration Method for Augmented Reality. In Proceedings of the Asia Pacific Computer Human Interaction, Shonan Village Center, Kanagawa, Japan, 15-17 July 1998.

24. Fiala, M. ARTag, a fiducial marker system using digital techniques. In Proceedings of the IEEE Computer Society Conference on Computer Vision \& Pattern Recognition, San Diego, CA, USA, 20-25 June 2005.

25. Chang, Y.; He, Z. Research on underground pipeline augmented reality system based on ARToolKit. Comput. Appl. Eng. Educ. 2005, 29, 196-199.

26. Wagner, D.; Schmalstieg, D. Artoolkitplus for pose tracking on mobile devices. In Proceedings of the Computer Vision Winter Workshop, St. Lambrecht, Australia, 6-8 February 2007.

27. Garrido-Jurado, S.; Muñoz-Salinas, R.; Madrid-Cuevas, F.J.; Marín-Jiménez, M.J. Automatic generation and detection of highly reliable fiducial markers under occlusion. Pattern Recognit. 2014, 47, 2280-2292. [CrossRef]

28. Jin, R.; Jiang, J.; Qi, Y.; Lin, D.; Song, T. Drone Detection and Pose Estimation Using Relational Graph Networks. Sensors 2019, 19, 1479. [CrossRef]

29. Gao, X.S.; Hou, X.R.; Tang, J.; Cheng, H.F. Complete solution classification for the perspective-three-point problem. IEEE Trans. Pattern Anal. Mach. Intell. 2003, 25, 930-943. 
30. Kim, H.; Lee, D.; Oh, T.; Choi, H.-T.; Myung, H. A Probabilistic Feature Map-Based Localization System Using a Monocular Camera. Sensors 2015, 15, 21636-21659. [CrossRef] [PubMed]

31. Lepetit, V.; Moreno-Noguer, F.; Fua, P. Epnp: An accurate o(n) solution to the pnp problem. Int. J. Comput. Vis. 2009, 81, 155-166. [CrossRef]

32. Abdel-Aziz, Y.I.; Karara, H.M. Direct linear transformation from comparator coordinates into object space coordinates in close-range photogrammetry. Photogramm. Eng. Remote Sens. 2015, 81, 103-107. [CrossRef]

33. Zhou, J.; Shang, Y.; Zhang, X.; Yu, W. A Trajectory and Orientation Reconstruction Method for Moving Objects Based on a Moving Monocular Camera. Sensors 2015, 15, 5666-5686. [CrossRef]

34. Tørdal, S.S.; Hovland, G. Relative Vessel Motion Tracking Using Sensor Fusion, Aruco Markers, and MRU Sensors. Model. Identif. Control 2017, 38, 79-93. [CrossRef]

35. Otsu, N. A Threshold Selection Method from Gray-Level Histograms. IEEE Trans. Syst. Man Cybern. 1979, 9, 62-66. [CrossRef]

36. Romero-Ramirez, F.; Munoz-Salinas, R.; Medina-Carnicer, R. Fractal Markers: A New Approach for Long-Range Marker Pose Estimation under Occlusion. 2019. Available online: https://www.researchgate.net/publication/332727382_Fractal_Markers_a_new_approach_for_long-range_ marker_pose_estimation_under_occlusion (accessed on 20 October 2019). [CrossRef]

37. Moré, J.J. The Levenberg-Marquardt algorithm: Implementation and theory. Lect. Notes Math. 1978, 630, 105-116.

38. Pestana, J.; Mellado-Bataller, I.; Sanchez-Lopez, J.L.; Fu, C.; Mondragon, I.F.; Campoy, P. A General Purpose Configurable Controller for Indoors and Outdoors GPS-Denied Navigation for Multirotor Unmanned Aerial Vehicles. J. Intell. Robot. Syst. 2014, 73, 387-400. [CrossRef]

39. Pixhawk. Available online: http://www.pixhawk.com/ (accessed on 26 March 2019).

40. Mavros. Available online: https://github.com/mavlink/mavros/ (accessed on 4 May 2019).

41. Ros. Available online: http://www.ros.org/ (accessed on 6 June 2019).

42. Optitrack. Available online: http://www.optitrack.com/ (accessed on 6 June 2019).

(C) 2019 by the authors. Licensee MDPI, Basel, Switzerland. This article is an open access article distributed under the terms and conditions of the Creative Commons Attribution (CC BY) license (http://creativecommons.org/licenses/by/4.0/). 